\title{
INEQUALITIES FOR THE SCHMIDT NUMBER OF BIPARTITE STATES
}

\author{
D. CARIELLO
}

\begin{abstract}
In this short note we show two completely opposite methods of constructing entangled states. Given a bipartite state $\gamma \in M_{k} \otimes M_{k}$, define $\gamma_{S}=(I d+F) \gamma(I d+F), \gamma_{A}=(I d-F) \gamma(I d-F)$, where $F \in M_{k} \otimes M_{k}$ is the flip operator. In the first method, entanglement is a consequence of the inequality $\operatorname{rank}\left(\gamma_{S}\right)<\sqrt{\operatorname{rank}\left(\gamma_{A}\right)}$. In the second method, there is no correlation between $\gamma_{S}$ and $\gamma_{A}$. These two methods show how diverse is quantum entanglement.

We prove that any bipartite state $\gamma \in M_{k} \otimes M_{k}$ satisfies

$$
S N(\gamma) \geq \max \left\{\frac{\operatorname{rank}\left(\gamma_{L}\right)}{\operatorname{rank}(\gamma)}, \frac{\operatorname{rank}\left(\gamma_{R}\right)}{\operatorname{rank}(\gamma)}, \frac{S N\left(\gamma_{S}\right)}{2}, \frac{S N\left(\gamma_{A}\right)}{2}\right\},
$$

where $S N(\gamma)$ stands for the Schmidt number of $\gamma$ and $\gamma_{L}, \gamma_{R}$ are the marginal states of $\gamma$.

We also present a family of PPT states in $M_{k} \otimes M_{k}$, whose members have Schmidt number equal to $n$, for any given $1 \leq n \leq\left\lceil\frac{k-1}{2}\right]$. This is a new contribution to the open problem of finding the best possible Schmidt number for PPT states.
\end{abstract}

\section{INTRODUCTION}

The separability problem in Quantum Information Theory asks for a deterministic criterion to distinguish the entangled states from the separable states [3]. This problem is known to be a hard problem even for bipartite mixed states [4,5].

The Schmidt number of a state $(S N(\gamma)$ - Definition 2.1) is a measure of how entangled a state is [10,11. If its Schmidt number is 1 then the state is separable. If its Schmidt number is greater than 1 then the state is entangled. A method to compute the Schmidt Number is unknown.

Denote by $M_{k}$ the set of complex matrices of order $k$. The separability problem has been completely solved in $M_{2} \otimes M_{2}$. A state in $M_{2} \otimes M_{2}$ is separable if and only if it is positive under partial transposition or simply PPT (Definition 2.1) 6, 9]. Therefore, the Schmidt number of a PPT state in $M_{2} \otimes M_{2}$ is equal to 1. Recently, the Schmidt number of every PPT state of $M_{3} \otimes M_{3}$ has been proved to be less or equal to 2 [2,12.

The authors of [8] left an open problem to determine the best possible Schmidt number for PPT states. They also presented a construction of PPT states in $M_{k} \otimes M_{k}$ whose Schmidt numbers are greater or equal to $\left\lceil\frac{k-1}{4}\right\rceil$. This was the first explicit example of a family of PPT states achieving a Schmidt number that scales linearly in the local dimension.

We investigate this matter. We present an explicit construction of PPT states in $M_{k} \otimes M_{k}$, whose Schmidt numbers are equal to $n$, for any given $1 \leq n \leq\left\lceil\frac{k-1}{2}\right\rceil$. This is a new contribution to their open problem.

We manage to compute the Schmidt number of these PPT states using the following inequality

$$
S N(\gamma) \geq \max \left\{\frac{S N\left(\gamma_{S}\right)}{2}, \frac{S N\left(\gamma_{A}\right)}{2}\right\},
$$

where $\gamma_{S}=(I d+F) \gamma(I d+F), \gamma_{A}=(I d-F) \gamma(I d-F)$ and $F \in M_{k} \otimes M_{k}$ is the flip operator (i.e., $F(a \otimes b)=b \otimes a$, for every $\left.a, b \in \mathbb{C}^{k}\right)$.

We believe this is one of the simplest constructions of an entangled PPT state made so far. 
Another inequality that we present here extends a result that was previously known for separable states ([7, Theorem 1]) to every state of $M_{k} \otimes M_{m}$. Denote by $\gamma_{L}$ and $\gamma_{R}$ the marginal states of a state $\gamma \in M_{k} \otimes M_{m}$ (Definition 2.1).

We show that every state $\gamma$ of $M_{k} \otimes M_{m}$ satisfies

$$
\operatorname{rank}(\gamma) S N(\gamma) \geq \max \left\{\operatorname{rank}\left(\gamma_{L}\right), \operatorname{rank}\left(\gamma_{R}\right)\right\}
$$

We can use this inequality to obtain a lower bound for the Schmidt number of low rank states.

Next, through a series of very technical results, the author of [1] obtained the following lower bounds for the $\operatorname{rank}\left(\gamma_{S}\right)$ of any separable state $\gamma \in M_{k} \otimes M_{k}$

$$
\operatorname{rank}\left(\gamma_{S}\right) \geq \max \left\{\frac{r}{2}, \frac{2}{r} \operatorname{rank}\left(\gamma_{A}\right)\right\},
$$

where $r$ is the marginal rank of $\gamma+F \gamma F$.

These inequalities can be combined into one inequality:

$$
\operatorname{rank}\left(\gamma_{S}\right) \geq \frac{2}{r} \operatorname{rank}\left(\gamma_{A}\right) \geq \frac{\operatorname{rank}\left(\gamma_{A}\right)}{\operatorname{rank}\left(\gamma_{S}\right)}
$$

Hence, $\operatorname{rank}\left(\gamma_{S}\right) \geq \sqrt{\operatorname{rank}\left(\gamma_{A}\right)}$ for every separable state $\gamma \in M_{k} \otimes M_{k}$. Therefore, if $\operatorname{rank}\left(\gamma_{S}\right)<$ $\sqrt{\operatorname{rank}\left(\gamma_{A}\right)}$ then $\gamma$ is entangled.

Next, we can combine equations 1.1 and 1.2 in order to obtain

$$
S N(\gamma) \geq \frac{\operatorname{rank}\left(\left(\gamma_{S}\right)_{L}\right)}{2 \operatorname{rank}\left(\gamma_{S}\right)} \text { and } S N(\gamma) \geq \frac{\operatorname{rank}\left(\left(\gamma_{A}\right)_{L}\right)}{2 \operatorname{rank}\left(\gamma_{A}\right)}
$$

We can easily create entangled states by satisfying $\frac{\operatorname{rank}\left(\left(\gamma_{S}\right)_{L}\right)}{\operatorname{rank}\left(\gamma_{S}\right)}>2$ or $\frac{\operatorname{rank}\left(\left(\gamma_{A}\right)_{L}\right)}{\operatorname{rank}\left(\gamma_{A}\right)}>2$ and no correlation between $\gamma_{S}$ and $\gamma_{A}$ is required.

These two methods of creating entangled states are completely opposite. One depends on a correlation between $\gamma_{S}, \gamma_{A}$ and the other does not. They show how diverse is quantum entanglement.

This paper is organized as follows.

- In Section II, we prove that $S N(\gamma) \geq \max \left\{\frac{S N\left(\gamma_{S}\right)}{2}, \frac{S N\left(\gamma_{A}\right)}{2}\right\}$ (Proposition 2.2) and we construct a PPT state whose Schmidt number is equal to $n$, for any given $n \in\left\{1, \ldots,\left\lceil\frac{k-1}{2}\right\rceil\right\}$ (Proposition 2.3).

- In Section III, we prove our main inequality $\operatorname{rank}(\gamma) S N(\gamma) \geq \max \left\{\operatorname{rank}\left(\gamma_{L}\right), \operatorname{rank}\left(\gamma_{R}\right)\right\}$ (Theorem 3.1) and two corollaries $S N(\gamma) \geq \frac{\operatorname{rank}\left(\left(\gamma_{S}\right)_{L}\right)}{2 \operatorname{rank}\left(\gamma_{S}\right)}$ and $S N(\gamma) \geq \frac{\operatorname{rank}\left(\left(\gamma_{A}\right)_{L}\right)}{2 \operatorname{rank}\left(\gamma_{A}\right)}$ (Corollaries 3.2 and 3.3 .

Notation: Given $x \in \mathbb{R}$, define $\lceil x\rceil=\min \{n \in \mathbb{Z}, n \geq x\}$. Identify $M_{k} \otimes M_{m} \simeq M_{k m}$ and $\mathbb{C}^{k} \otimes \mathbb{C}^{m} \simeq \mathbb{C}^{k m}$ via Kronecker product. Let us call a positive semidefinite Hermitian matrix of $M_{k m}$ a (non-normalized bipartite) state of $M_{k} \otimes M_{m}$. Let $\Im(\delta)$ denote the image of $\delta \in M_{k} \otimes M_{m}$ within $\mathbb{C}^{k} \otimes \mathbb{C}^{m}$. Given $w \in \mathbb{C}^{k} \otimes \mathbb{C}^{m}$ denote by $S R(w)$ its Schmidt rank (or tensor rank). Let the trace of a matrix $A \in M_{k}$ be denoted by $\operatorname{tr}(A)$. 


\section{Preliminary Inequalities}

In this section we present two preliminary inequalities (Proposition 2.2). They have independent interest as we can see in Proposition 2.3. There we construct a family of PPT states in $M_{k} \otimes M_{k}$ whose members have Schmidt number equal to $n$, for any given $1 \leq n \leq\left\lceil\frac{k-1}{2}\right\rceil$.

Definition 2.1. Given a state $\delta=\sum_{i=1}^{n} A_{i} \otimes B_{i} \in M_{k} \otimes M_{m}$, define

- the Schmidt number of $\delta$ as

$S N(\delta)=\min \left\{\max _{j}\left\{S R\left(w_{j}\right)\right\}, \delta=\sum_{j=1}^{m} w_{j}{\overline{w_{j}}}^{t}\right\}$ (This minimum is taken over all decompositions of $\delta$ as $\left.\sum_{j=1}^{m} w_{j} \bar{w}_{j}^{t}\right)$.

- the partial transposition of $\delta$ as $\delta^{\Gamma}=\sum_{i=1}^{n} A_{i} \otimes B_{i}^{t}$. Moreover, let us say that $\delta$ is positive under partial transposition or simply a PPT state if and only if $\delta$ and $\delta^{\Gamma}$ are states.

- the marginal states of $\delta$ as $\delta_{L}=\sum_{i=1}^{n} A_{i} \operatorname{tr}\left(B_{i}\right)$ and $\delta_{R}=\sum_{i=1}^{n} B_{i} \operatorname{tr}\left(A_{i}\right)$.

Proposition 2.2. Every state $\gamma \in M_{k} \otimes M_{k}$ satisfies $S N(\gamma) \geq \max \left\{\frac{S N\left(\gamma_{S}\right)}{2}, \frac{S N\left(\gamma_{A}\right)}{2}\right\}$.

Proof. By definition 2.1, there is a subset $\left\{w_{1}, \ldots, w_{n}\right\} \subset \mathbb{C}^{k} \otimes \mathbb{C}^{k}$ such that $\gamma=\sum_{i=1}^{n} w_{i} \bar{w}_{i}^{t}$ and $S R\left(w_{i}\right) \leq S N(\gamma)$, for every $i$.

Therefore, $(I d \pm F) \gamma(I \pm F)=\sum_{i=1}^{n} v_{i} \bar{v}_{i}^{t}$, where $v_{i}=(I d \pm F) w_{i}$. Notice that, for every $i$,

$$
S R\left(v_{i}\right)=S R\left(w_{i} \pm F w_{i}\right) \leq 2 S R\left(w_{i}\right) \leq 2 S N(\gamma) .
$$

Hence, $S N((I d \pm F) \gamma(I \pm F)) \leq 2 S N(\gamma)$.

Proposition 2.3. Let $v=\sum_{i=1}^{n} a_{i} \otimes b_{i}$, where $\left\{a_{1}, \ldots, a_{n}, b_{1}, \ldots b_{n}\right\}$ is a linearly independent subset of $\mathbb{C}^{k}$. Define

$$
\gamma=I d+F+\epsilon\left(v \bar{v}^{t}\right) \in M_{k} \otimes M_{k} .
$$

(1) For every $\epsilon>0, S N(\gamma)=n$. Notice that $1 \leq n \leq\left\lceil\frac{k-1}{2}\right\rceil$.

(2) There is $\epsilon>0$ such that $\gamma$ is positive under partial transposition.

Proof. (1) Notice that $\gamma_{A}=(I d-F) \gamma(I d-F)=\epsilon\left(a \bar{a}^{t}\right)$, where $a=\sum_{i=1}^{n} a_{i} \otimes b_{i}-b_{i} \otimes a_{i}$.

Since $\left\{a_{1}, \ldots, a_{n}, b_{1}, \ldots b_{n}\right\}$ is linearly independent then $S R(v)=n$ and $S R(a)=2 n$. Hence,

$$
S N\left(\epsilon\left(v \bar{v}^{t}\right)\right)=S R(v)=n \text { and } S N\left(\gamma_{A}\right)=S R(a)=2 n .
$$

Thus, $S N(\gamma) \geq \frac{S N\left(\gamma_{A}\right)}{2}=n$, by Proposition 2.2.

Next, the separability of $I d+F \in M_{k} \otimes M_{k}$ is a well known fact, therefore $S N(I d+F)=1$.

Finally, $S N(\gamma) \leq \max \left\{S N(I d+F), S N\left(\epsilon\left(v \bar{v}^{t}\right)\right)\right\}=\max \{1, n\}=n$. Therefore, $S N(\gamma)=n$.

(2) Notice that $(I d+F)^{\Gamma}=I d+u u^{t}$, where $u=\sum_{i=1}^{k} e_{i} \otimes e_{i}$ and $\left\{e_{1}, \ldots, e_{k}\right\}$ is the canonical basis of $\mathbb{C}^{k}$. So $(I d+F)^{\Gamma}$ is positive definite and, for a small $\epsilon,(I d+F)^{\Gamma}+\epsilon\left(v \bar{v}^{t}\right)^{\Gamma}$ is positive definite too. 


\section{Main Inequality}

In this section, we present our main result (Theorem 3.1) and two corollaries (Corollaries 3.2 and 3.3 ).

Theorem 3.1. If $\gamma \in M_{k} \otimes M_{m}$ is a state then $\operatorname{rank}(\gamma) S N(\gamma) \geq \max \left\{\operatorname{rank}\left(\gamma_{L}\right), \operatorname{rank}\left(\gamma_{R}\right)\right\}$.

Proof. The proof is an induction on $\operatorname{rank}(\gamma)$. The case $\operatorname{rank}(\gamma)=0$ is trivial. If $\operatorname{rank}(\gamma)=1$ then $S N(\gamma)=\operatorname{rank}\left(\gamma_{L}\right)=\operatorname{rank}\left(\gamma_{R}\right)$.

Let $\operatorname{rank}(\gamma)>1$ and assume that this result is valid for states $\delta \in M_{k} \otimes M_{m}$ satisfying $\operatorname{rank}(\delta)<\operatorname{rank}(\gamma)$.

Since $\Im(\gamma) \subset \Im\left(\gamma_{L} \otimes \gamma_{R}\right)$ then $\gamma$ can be embedded in $M_{\operatorname{rank}\left(\gamma_{L}\right)} \otimes M_{\operatorname{rank}\left(\gamma_{R}\right)}$. The embedding does not change its rank or its Schmidt number. Thus, we can assume without loss of generality that $\operatorname{rank}\left(\gamma_{L}\right)=k$ and $\operatorname{rank}\left(\gamma_{R}\right)=m$.

Let $v \in \Im(\gamma) \backslash\{0\}$ be such that $S R(v)=S N(\gamma)$.

- If $k \geq m$ then choose $U \in M_{k}$ satisfying $\operatorname{rank}(U)=k-S N(\gamma)$ and $(U \otimes I d) v=0$. Define $\delta=(U \otimes I d) \gamma\left(U^{*} \otimes I d\right)$. Note that $\operatorname{rank}(\delta) \leq \operatorname{rank}(\gamma)-1$, since $\Im(\delta) \subset(U \otimes I d)(\Im(\gamma))$ and $(U \otimes I d) v=0$.

- If $k<m$ then choose $U \in M_{m}$ satisfying $\operatorname{rank}(U)=m-S N(\gamma)$ and $(I d \otimes U) v=0$. Define $\delta=(I d \otimes U) \gamma\left(I d \otimes U^{*}\right)$. Note that $\operatorname{rank}(\delta) \leq \operatorname{rank}(\gamma)-1$, since $\Im(\delta) \subset(I d \otimes U)(\Im(\gamma))$ and $(I d \otimes U) v=0$.

In any case, by induction hypothesis, $\operatorname{rank}(\delta) S N(\delta) \geq \max \left\{\operatorname{rank}\left(\delta_{L}\right), \operatorname{rank}\left(\delta_{R}\right)\right\}$.

- If $k \geq m$ then $\delta_{L}=U \gamma_{L} U^{*}$. Since $\gamma_{L}$ is positive definite then $\operatorname{rank}\left(\delta_{L}\right)=\operatorname{rank}(U)=$ $k-S N(\gamma)$.

- If $k<m$ then $\delta_{R}=U \gamma_{R} U^{*}$. Since $\gamma_{R}$ is positive definite then $\operatorname{rank}\left(\delta_{R}\right)=\operatorname{rank}(U)=$ $m-S N(\gamma)$.

Since $\operatorname{rank}(\delta) \leq \operatorname{rank}(\gamma)-1$ and $S N(\delta) \leq S N(\gamma)$ then

- $(\operatorname{rank}(\gamma)-1) S N(\gamma) \geq k-S N(\gamma)$, if $k \geq m$. Therefore, $\operatorname{rank}(\gamma) S N(\gamma) \geq k$.

- $(\operatorname{rank}(\gamma)-1) S N(\gamma) \geq m-S N(\gamma)$, if $k<m$. Therefore, $\operatorname{rank}(\gamma) S N(\gamma) \geq m$.

The induction is complete.

Corollary 3.2. If $\gamma \in M_{k} \otimes M_{k}$ is a state then $S N(\gamma) \geq \frac{\operatorname{rank}\left(\left(\gamma_{A}\right)_{L}\right)}{2 \operatorname{rank}\left(\gamma_{A}\right)}$.

Proof. First, notice that $\left(\gamma_{A}\right)_{L}=\left(\gamma_{A}\right)_{R}$. Therefore, $\operatorname{rank}\left(\left(\gamma_{A}\right)_{L}\right)=\operatorname{rank}\left(\left(\gamma_{A}\right)_{R}\right)$. 
Next, since $S N\left(\gamma_{A}\right) \leq 2 S N(\gamma)$, by Proposition 2.2, then $\operatorname{rank}\left(\gamma_{A}\right) S N(\gamma) \geq \frac{1}{2} \operatorname{rank}\left(\gamma_{A}\right) S N\left(\gamma_{A}\right) \geq$ $\frac{\operatorname{rank}\left(\left(\gamma_{A}\right)_{L}\right)}{2}$, by Theorem 3.1 .

Corollary 3.3. If $\gamma \in M_{k} \otimes M_{k}$ is a state then $S N(\gamma) \geq \frac{\operatorname{rank}\left(\left(\gamma_{S}\right)_{L}\right)}{2 \operatorname{rank}\left(\gamma_{S}\right)}$.

Proof. First, notice that $\left(\gamma_{S}\right)_{L}=\left(\gamma_{S}\right)_{R}$. Therefore, $\operatorname{rank}\left(\left(\gamma_{S}\right)_{L}\right)=\operatorname{rank}\left(\left(\gamma_{S}\right)_{R}\right)$.

Since $S N\left(\gamma_{S}\right) \leq 2 S N(\gamma)$, by Proposition [2.2, then $\operatorname{rank}\left(\gamma_{S}\right) S N(\gamma) \geq \frac{1}{2} \operatorname{rank}\left(\gamma_{S}\right) S N\left(\gamma_{S}\right) \geq$ $\frac{\operatorname{rank}\left(\left(\gamma_{S}\right)_{L}\right)}{2}$, by Theorem 3.1.

\section{Summary AND Conclusion}

We presented an inequality that relates the marginal ranks of any bipartite state of $M_{k} \otimes M_{m}$ to its rank and its Schmidt number. Using this inequality, we described a method of constructing entangled states which is not based on any correlation between $\operatorname{rank}\left(\gamma_{A}\right)$ and $\operatorname{rank}\left(\gamma_{S}\right)$. This form of entanglement differs completely from the entanglement derived from the inequality $\operatorname{rank}\left(\gamma_{S}\right)<$ $\sqrt{\operatorname{rank}\left(\gamma_{A}\right)}$. We also constructed a family of PPT states whose members have Schmidt number equal to $n$, for any given $1 \leq n \leq\left\lceil\frac{k-1}{2}\right\rceil$. This is a new contribution to the open problem of finding the best possible Schmidt number for PPT states.

\section{REFERENCES}

[1] D. Cariello, A gap for PPT entanglement, Linear Algebra and its Applications 529 (2017), 89-114.

[2] L. Chen, Y. Yang, and W. S. Tang, Schmidt number of bipartite and multipartite states under local projections, Quantum Information Processing 16 (2017), no. 3, 75.

[3] O. Gühne and G Tóth, Entanglement detection, Physics Reports 474 (2009), no. 1-6, 1-75.

[4] L. Gurvits, Classical deterministic complexity of Edmonds' Problem and quantum entanglement, Proceedings of the thirty-fifth annual ACM symposium on Theory of computing; Jun 9-11; San Diego, CA, USA. New York: ACM press, 2003, pp. 10-19.

[5] Classical complexity and quantum entanglement, Journal of Computer and System Sciences 69 (2004), no. $3,448-484$.

[6] M. Horodecki, P. Horodecki, and R. Horodecki, Separability of mixed states: necessary and sufficient conditions, Phys. Lett. A. 223 (1996), 1-8.

[7] P. Horodecki, J. A. Smolin, B. M. Terhal, and A. V. Thapliyal, Rank two bipartite bound entangled states do not exist, Theoretical Computer Science 292 (2003), no. 3, 589-596.

[8] M. Huber, L. Lami, C. Lancien, and A. Müller-Hermes, High-dimensional entanglement in states with positive partial transposition., arXiv:1802.04975 (2018).

[9] A. Peres, Separability criterion for density matrices, Physical Review Letters 77 (1996), no. 8, 1413.

[10] J. Sperling and W. Vogel, The Schmidt number as a universal entanglement measure., Physica Scripta 83 (2011), no. 4, 045002.

[11] B. M. Terhal and P. Horodecki, Schmidt number for density matrices, Phys. Rev. A 61 (2000), no. 4, 040301.

[12] Y. Yang, D. H. Leung, and W. S. Tang, All 2-positive linear maps from M3 (C) to M3 (C) are decomposable, Linear Algebra and its Applications 503 (2016), 233-247.

Faculdade de Matemática,

Universidade Federal de Uberlândia, 38.400-902 UBERlÂNDIA, BRAZIL.

E-mail address: dcariello@ufu.br 